\title{
Aspectos relacionados ao diagnóstico e tratamento da hanseníase: uma revisão sistemática
}

\author{
Aspects related to the diagnosis and treatment of leprosy: a systematic review \\ Aspectos relacionados com el diagnóstico y el tratamiento de la lepra: una revisión \\ sistemática
}

Eduarda Rêgo Saraiva ${ }^{1 *}$, Dandara dos Santos Gomes Gadelha ${ }^{1}$, Siana Malena Soares Brito ${ }^{1}$, André Augusto Guerra Gomes' ${ }^{1}$ Ingrid Beatriz Galdino Soares ${ }^{1}$, Murilo Rodrigues da Silva ${ }^{1}$, Ana Rachel Oliveira de Andrade ${ }^{1}$, Vanessa de Sousa do Vale ${ }^{1}$, Tereza Cristina de Carvalho Souza Garcês ${ }^{1}$.

\section{RESUMO}

Objetivo: Identificar as principais formas de diagnóstico e analisar os fatores associados a adesão e abandono do tratamento da hanseníase no Brasil. Métodos: Revisão sistemática de literatura. A busca foi realizada nas bases de dados Scientific Electronic Library Online (Scielo), Literatura Latino-Americana e do Caribe em Ciências da Saúde (LILACS) e Medical Literature Analysis and Retrieval System Online (Medline), sendo selecionadas publicações no período de 2010 a 2020. Utilizaram-se os descritores: saúde púbica and diagnóstico and quimioterapia combinada and hanseníase. Resultados: Verificou-se a importância do diagnóstico precoce na diminuição do processo de transmissibilidade. Sobre à adesão, foram relatadas: relevância da educação em saúde, papel da religiosidade e dos vínculos familiares para o enfrentamento do tratamento. Fatores sociodemográficos, dificuldades de compreensão das orientações da equipe de saúde, elementos físicos, discriminação, impotência diante do problema foram influenciadores do abandono do tratamento. Considerações finais: A hanseníase e os aspectos relacionados à adesão/abandono do tratamento representam um desafio a ser superado pela saúde pública Brasileira. Espera-se que o resultado dessa pesquisa seja elemento norteador para futuros trabalhos sobre o tema. Sugere-se que, futuramente, sejam realizados estudos com o intuito de investigar a realidade local, em relação aos dados sobre adesão, abandono e formas de diagnóstico da hanseníase.

Palavras-chave: Saúde pública, Poliquimioterapia, Hanseníase.

\begin{abstract}
Objective: Identify the main forms of diagnosis and analyze the factors associated with adherence and abandonment of leprosy treatment in Brazil. Methods: Systematic literature review. The search was carried out in the databases Scientific Electronic Library Online (Scielo), Literatura Latino-Americana e do Caribe em Ciências da Saúde (LILACS) and Medical Literature Analysis and Retrieval System Online (Medline), publications were selected from 2010 to 2020. The descriptors: public health and diagnosis and combined chemotherapy and leprosy were used. Results: The importance of early diagnosis was found to decrease the transmissibility process. Regarding adherence, the following were reported: the relevance of health education, the role of religiosity and family ties for coping with treatment. Sociodemographic factors, difficulties in understanding the guidelines of the health team, physical elements, discrimination, impotence in the face of the problem were influencers of treatment abandonment. Final considerations: Leprosy and aspects related to treatment adherence/abandonment represent a challenge to be overcome by Brazilian public health. It is expected that the result of this research will be a guiding element for future work on the topic. It is suggested that, in the future, studies be carried out in order to investigate the local reality, in relation to data on adherence, abandonment and forms of diagnosis of leprosy.
\end{abstract}

Keywords: Public health, Drug therapy combination, Leprosy.

${ }^{1}$ Faculdade de Ciências Humanas, Exatas e da Saúde do Piauí / Instituto de Educação Superior do Vale do
Parnaíba (FAHESP/IESVAP), Parnaíba - PI. *E-mail: eduardarego@hotmail.com SUBMETIDO EM: 7/2020

PUBLICADO EM: 12/2020 


\section{RESUMEN}

Objetivo: Identificar las principales formas de diagnóstico y analizar los factores asociados con la adherencia y el abandono del tratamiento de la lepra en Brasil. Métodos: Revisión sistemática de la literatura. La búsqueda se realizó en las bases de datos. Scientific Electronic Library Online (Scielo), Literatura LatinoAmericana e do Caribe em Ciências da Saúde (LILACS) y Medical Literature Analysis and Retrieval System Online (Medline), se seleccionaron publicaciones de 2010 a 2020. Los descriptores: salud pública and diagnóstico and quimioterapia combinada and lepra fueron utilizados. Resultados: Se descubrió que la importancia del diagnóstico precoz disminuye el proceso de transmisibilidad. Con respecto a la adherencia, se informó lo siguiente: la relevancia de la educación para la salud, el papel de la religiosidad y los lazos familiares para hacer frente al tratamiento. Los factores sociodemográficos, las dificultades para comprender las pautas del equipo de salud, los elementos físicos, la discriminación, la impotencia ante el problema influyeron en el abandono del tratamiento. Consideraciones finales: La lepra y los aspectos relacionados con el cumplimiento/abandono del tratamiento representan un desafío que la salud pública brasileña debe superar. Se espera que el resultado de esta investigación sea un elemento guía para el trabajo futuro sobre el tema. Se sugiere que, en el futuro, se realicen estudios para investigar la realidad local, en relación con los datos sobre adherencia, abandono y formas de diagnóstico de lepra.

Palabras clave: Salud pública, Quimioterapia combinada, Lepra.

\section{INTRODUÇÃO}

A hanseníase é uma das doenças mais antigas da humanidade (MELÃO S, et al., 2011; ROSA SPS, et al., 2016). As referências mais remotas datam de 600 a.C. e procedem da Ásia, que junto com a África, são consideradas o berço da doença (BOECHAT N, 2012, PINHEIRO LCS, 2017; LAMANO LR, 2013). Entretanto, o termo hanseníase é uma iniciativa brasileira para minimizar o preconceito secular atribuído à doença, adotado pelo Ministério da Saúde em 1976. Com isso, o nome Lepra e seus adjetivos passaram a ser proibidos no país (BRASIL MS, 2016; CARVALHO KA, 2015).

De acordo com o Ministério da Saúde, a hanseníase é considerada uma doença infectocontagiosa, crônica e granulomatosa causada pelo Mycobacterium leprae, frequentemente conhecido como bacilo de Hansen (BRASIL MS, 2016). Este é um Bacilo Álcool-Ácido Resistente (BAAR) que ao infectar o organismo humano, tem tropismo pelas células de schwann, que são células do sistema nervoso periférico, resultando em comprometimento da função neural periférica e manifestando-se através de sinais dermatoneurológicos (ARAUJO AERA, et al., 2014, PAZ BL, et al., 2018).

De acordo com a Organização Mundial da Saúde OMS (2012), ao considerar o continente americano, o Brasil ocupa a primeira posição, com cerca de 33.955 casos novos.

O Brasil ocupa a segunda posição do mundo, entre os países que registram novos casos (BRASIL MS, 2016). Em razão da elevada carga bacteriológica, a doença permanece como um importante problema de saúde pública no país (RIBEIRO MDA, 2018). Segundo Silva DRX, et al. (2010), a hanseníase é considerada uma doença contagiosa que quando não é tratada traz consigo o estigma negativo e atitudes preconceituosa de rejeição e discriminação aos portadores da doença, sendo este, normalmente, excluído da sociedade.

Em relação à discriminação sofrida pelos doentes, Eidt LM (2004) destaca que nos tempos antigos, as pessoas com hanseníase também eram proibidas de comerem ou dormirem com pessoas sadias e casarem com cônjuges que não fossem doentes. Ainda nessa época, como não havia cura, as maiorias desenvolviam deformações e morriam. A hanseníase pode atingir indivíduos inseridos em qualquer classe social, mas a sua incidência é maior nas classes mais baixas da população, devido à presença de condições socioeconômicas desfavoráveis e condições precárias de vida e saúde, o que facilita a contaminação e a propagação do bacilo causador dessa enfermidade (LOPES VAS, RANGELEM, 2014).

O diagnóstico da doença é essencialmente clínico e epidemiológico, realizado por meio do exame geral e dermatoneurólogico para identificar lesões ou áreas da pele com alteração de sensibilidade e/ou comprometimento de nervos periféricos, com alterações sensitivas, motoras ou autonômicas. 
Quanto ao tratamento, utilizam-se os esquemas de poliquimioterapia (PQT) recomendados pela Organização Mundial de Saúde (OMS), que se cumpridos rigorosamente levam a recuperação (RAMOS JMH, SOUTO FJD, 2010). A PQT é constituída pela associação de dapsona, clofazimina e rifampicina, sendo o tratamento concluído entre seis e nove meses dependendo da classificação da doença (BRASIL MS, 2008).

A escolha dos medicamentos dependerá da classificação da doença que pode ser subdividida em paucibacilar e multibacilar (PEREIRA SVM, et al., 2008). Mesmo com sólidas evidências sobre a comprovada eficácia da PQT, ainda há casos de hanseníase que ficam sem tratamento, ou seja, são interrompidos. Isso pode estar relacionado com fatores como a duração do tratamento, que dependendo da classificação da doença pode chegar até 18 meses, ou as reações raras que podem acometer algumas pessoas (BRASIL MS, 2008).

Sabe-se que o abandono do tratamento implica em maior risco de transmissão dos casos bacilíferos detectados e não tratados adequadamente, assim, dá-se continuidade à cadeia de transmissão que havia sido interrompida com o início do tratamento medicamentoso, aumentando o risco de desenvolvimento de incapacidades físicas e deformidades e, por conseguinte, o estigma social e a discriminação contra as pessoas afetadas e suas famílias. Dessa forma, casos de abandono do tratamento em curso de um regime terapêutico constituem um grave problema para os profissionais da área de saúde pública e ao Programa de Controle da Hanseníase (ARAUJO AERA, et al., 2014).

Diante da realidade observada no contexto nacional e local, onde há um aumento da incidência da hanseníase e diante dos casos de abandono de tratamento, surgiu o interesse pelo tema por parte das autoras, que se puseram a indagar sobre a problemática da hanseníase no cenário atual e passaram, empiricamente, a buscar obter mais informações sobre o assunto e que culminou com o desejo de abordar a temática neste trabalho. Além disso, essa pesquisa visa chamar atenção para a importância da educação em saúde e conscientização do tratamento. Diante do exposto, o presente trabalho tem como objetivo identificar as principais formas de diagnóstico e analisar os fatores associados à adesão e abandono do tratamento da hanseníase no Brasil.

\section{MÉTODOS}

Este estudo caracteriza-se como uma revisão sistemática de literatura, que pode ser definido como um tipo de estudo secundário, os quais utilizam publicações de estudos primários como fonte de obtenção dos dados para a realização das análises. Um exemplo de estudo primário são os artigos científicos que relatam os resultados de determinadas pesquisas em primeira mão (GALVAO TF, PEREIRA MG, 2014).

A pesquisa foi operacionalizada por meio de seis etapas, as quais estão estreitamente interligadas: elaboração da pergunta norteadora, busca na literatura, coleta de dados, análise crítica dos estudos incluídos, discussão dos resultados e apresentação da revisão sistemática (SOUZA MT, et al., 2010).

A busca na literatura foi realizada principalmente nas bases de dados Scientific Eletronic Library online (SCIELO), Literatura Latino-americana e do Caribe em Ciências da Saúde (LILACS) e Medical Literature Analysis and Retrievel System Online (Medline), utilizando-se a combinação de descritores cadastrados nos Descritores em Ciências da Saúde (DECs): saúde púbica (public health), diagnóstico (diagnosis), quimioterapia combinada (drug therapy, combination) e hanseníase (leprosy), utilizando o operador booleano "and".

Foram utilizados como critérios de inclusão, artigos científicos originais que contemplassem a temática, publicados em língua portuguesa e inglesa, no período de 2010 a 2020. Consequentemente, foram excluídas as revisões sistemáticas de literatura, os trabalhos que estavam foram do recorte temporal estabelecido e aqueles que fugiram ao tema proposto.

A análise dos dados foi realizada de forma descritiva e os estudos foram reunidos, os quais permitiram avaliar as evidências, bem como identificar a necessidade de investigações futuras acerca da temática. Para a extração de dados, foram primeiramente avaliados os títulos dos artigos, a fim de verificar a relação com a temática. Em seguida, os resumos dos estudos foram analisados para se averiguar seus objetivos e metodologia. Por fim, o restante dos artigos foi apurado nos conformes dos critérios de inclusão e exclusão através de toda sua leitura e análise integralmente. 
A partir da combinação dos descritores foi realizada uma avaliação inicial por meio dos títulos e logo após verificou-se quais deles estavam fora dos critérios de inclusão. A Figura 1 apresenta a esquematização de como foi realizado o processo de seleção das publicações do estudo.

Figura 1 - Método de seleção dos estudos incluídos na revisão sistemática no período de 2010 a 2020. Parnaíba-PI, 2020.

Descritores: "Saúde pública" and "hanseníase" 17 resultados (MEDLINE=1;

$$
\text { LILACS=7; SCIELO=9) }
$$

Descritores: "Diagnóstico" and "Quimioterapia combinada" and "Hanseníase" = 36 resultados (MEDLINE=2; LILACS=23 e SCIELO=11)

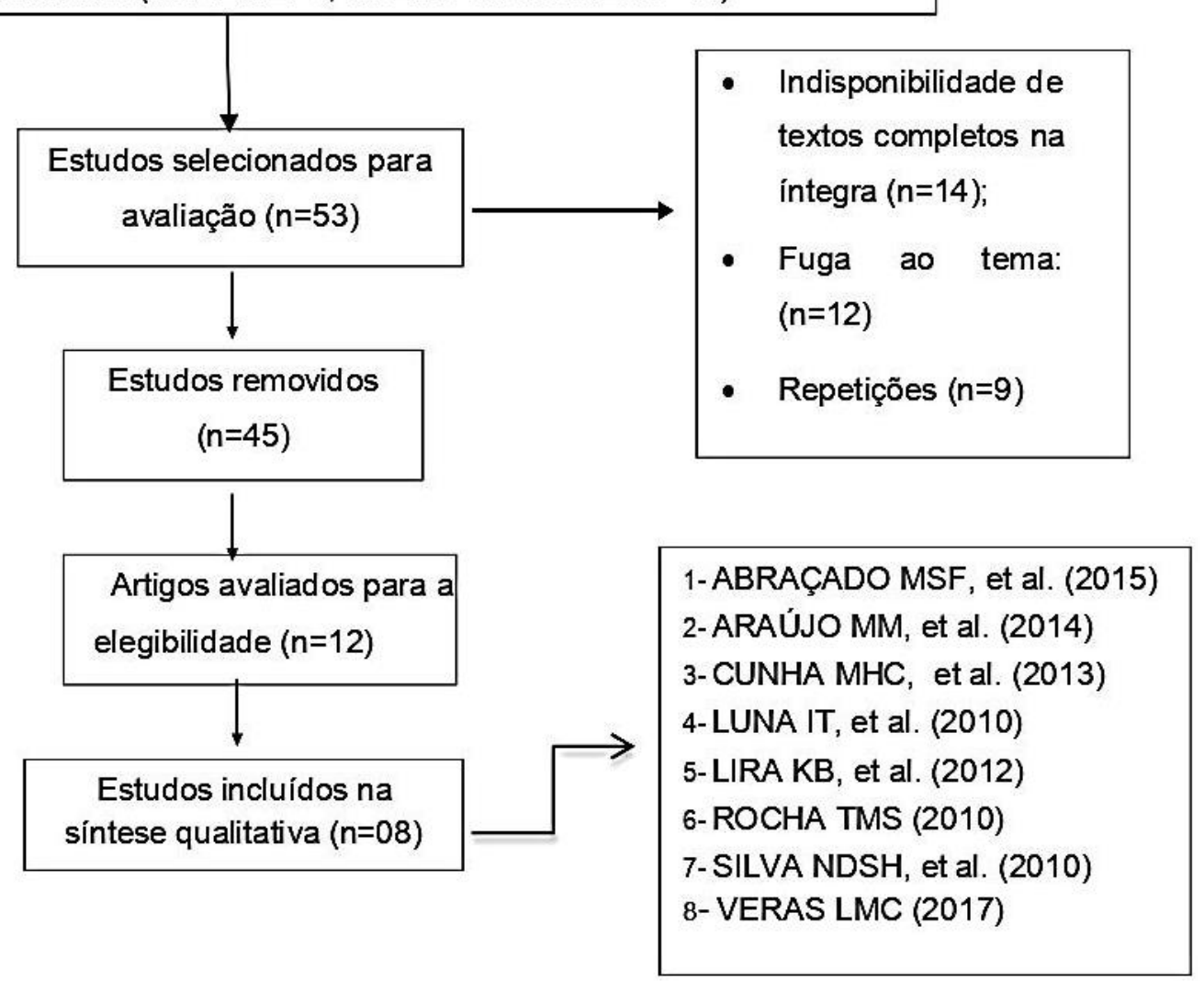

Fonte: Saraiva ER, et al., 2020.

\section{RESULTADOS E DISCUSSÃO}

Após a busca nas bases de dados foram identificados um total de oito artigos que atenderam ao objetivo proposto para nortear este trabalho, sendo a maior parte das pesquisas selecionadas foi publicada no ano de 2010. Além disso, a maioria das publicações selecionadas foi encontrada nas bases de dados Scielo e LILACS.

A publicação mais recente que atendeu aos critérios de seleção foi do ano de 2017. Buscou-se listar nos artigos fatores relacionados à forma de diagnóstico e que influenciam na adesão e abandono do tratamento, assim como pode ser observado no Quadro 1. 


\section{Revista Eletrônica Acervo Saúde / Electronic Journal Collection Health | ISSN 2178-2091}

Quadro 1 - Estudos utilizados na revisão sistemática no período de 2010-2020. Parnaíba - PI, 2020.

\begin{tabular}{|c|c|c|c|c|}
\hline Autor (ano) & Título & Forma de diagnóstico & Adesão & Abandono \\
\hline $\begin{array}{l}\text { Abraçado MSF, } \\
\text { et al. (2015) }\end{array}$ & $\begin{array}{l}\text { Adesão ao tratamento de } \\
\text { hanseníase e pacientes } \\
\text { com episódios reacionais } \\
\text { hansênicos em uma } \\
\text { unidade de referência }\end{array}$ & $\begin{array}{l}\text { Classificação de Madri: indeterminada } \\
\text { (I), dimorfa (D), tuberculoide }(T) \text { e } \\
\text { virchowiano }(V) \text { e do MS, por meio da } \\
\text { classificação operacional PB para os } \\
\text { paucibacilares e MB para os } \\
\text { multibacilares. }\end{array}$ & $\begin{array}{c}\text { Influência dos fatores } \\
\text { sociodemográficos na adesão ao } \\
\text { tratamento das reações hansênicas. } \\
\text { Maior tendência à adesão de } \\
\text { pacientes com formas } \\
\text { multibacilares, com tempo de } \\
\text { diagnóstico de hanseníase maior } \\
\text { que um ano, tipo reacional RR e } \\
\text { neurites. }\end{array}$ & $\begin{array}{l}\text { Predominância em pacientes do sexo } \\
\text { masculino. Baixo nível de escolaridade } \\
\text { e pouco nível de compreensão às } \\
\text { orientações repassadas. Quantidade } \\
\text { de drogas a ser ingerida é bem maior, } \\
\text { podendo ocorrer efeitos colaterais. }\end{array}$ \\
\hline $\begin{array}{l}\text { Araújo MM, et al. } \\
\qquad(2014)\end{array}$ & $\begin{array}{l}\text { Perfil clínico- } \\
\text { epidemiológico de } \\
\text { pacientes que } \\
\text { abandonaram o } \\
\text { tratamento de } \\
\text { hanseníase } \\
\end{array}$ & $\begin{array}{l}\text { Classificação do Ministério da Saúde: } \\
\text { Paucibacilar-PB e Multibacilar-MB. }\end{array}$ & $\begin{array}{l}\text { Mulheres e pacientes menores de } \\
\qquad 15 \text { anos }\end{array}$ & $\begin{array}{c}\text { Gênero masculino, de raça parda, em } \\
\text { idade produtiva, com baixa escola- } \\
\text { ridade, no início ou metade do } \\
\text { tratamento. }\end{array}$ \\
\hline $\begin{array}{l}\text { Cunha MHCM, et } \\
\text { al. (2013) }\end{array}$ & \begin{tabular}{|} 
Episódios reacionais \\
hansênicos: estudo de \\
fatores relacionados com \\
adesão ao tratamento \\
em uma unidade de \\
referência
\end{tabular} & $\begin{array}{l}\text { O diagnóstico clínico e histológico: segue } \\
\text { os padrões: I (indeterminada), TT } \\
\text { (Tuberculóide), BT (Boderline-Tubercu- } \\
\text { lóide), BB (borderline-borderline); BL } \\
\text { (borderline-lepro); LL (lepro- } \\
\text { lepromatoso). Os pacientes que } \\
\text { desenvolveram reação hansênica foram } \\
\text { classificados de acordo com os aspectos } \\
\text { clínicos apresentados como portadores } \\
\text { de reação tipo } 1 \text { e reação tipo } 2 \text {. }\end{array}$ & $\begin{array}{c}\text { Educação em saúde em nível } \\
\text { individual ou até mesmo com a } \\
\text { formação de um grupo de } \\
\text { autocuidados, no sentido de reforçar } \\
\text { a necessidade de um tratamento } \\
\text { sério por parte do paciente e de } \\
\text { acompanhamento contínuo. } \\
\text { Pacientes do sexo feminino } \\
\text { portadoras das formas clínica } \\
\text { paucibacilar. }\end{array}$ & $\begin{array}{l}\text { Sexo masculino, faixa etária entre } 25 \text { a } \\
45 \text { anos, solteiros, com ensino médio e } \\
\text { renda familiar igual ou maior que um } \\
\text { salário mínimo. Dor, desconforto físico, } \\
\text { alteração na aparência física e } \\
\text { sofrimento, além da grande dificuldade } \\
\text { ou impossibilidade de executar tarefas } \\
\text { domésticas. }\end{array}$ \\
\hline $\begin{array}{l}\text { Luna IT, et al. } \\
\qquad(2010)\end{array}$ & $\begin{array}{l}\text { Adesão ao tratamento da } \\
\text { Hanseníase: dificuldades } \\
\text { inerentes aos portadores }\end{array}$ & $\begin{array}{l}\text { Diagnóstico clínico preconizado pelo } \\
\text { ministério da saúde. }\end{array}$ & $\begin{array}{l}\text { Realização de visitas domiciliares e } \\
\text { prática de atividade de educação } \\
\text { em saúde proporcionaram uma } \\
\text { investigação com abordagem } \\
\text { holística, oferecida aos portadores } \\
\text { de hanseníase. }\end{array}$ & $\begin{array}{l}\text { Fragilidade na credibilidade por parte } \\
\text { do paciente quanto ao diagnóstico } \\
\text { médico e a não aceitação do uso de } \\
\text { PQT. As informações acerca da } \\
\text { importância da adesão ao tratamento } \\
\text { medicamentoso foram compreendidas } \\
\text { de maneira superficial. }\end{array}$ \\
\hline
\end{tabular}




\section{Revista Eletrônica Acervo Saúde / Electronic Journal Collection Health | ISSN 2178-2091}

\begin{tabular}{|c|c|c|c|c|}
\hline Autor (ano) & Título & Forma de diagnóstico & Adesão & Abandono \\
\hline $\begin{array}{l}\text { Lira KB, et al. } \\
\qquad(2012)\end{array}$ & $\begin{array}{l}\text { Knowledge of the } \\
\text { patients regarding } \\
\text { leprosy and adherence }\end{array}$ & $\begin{array}{c}\text { Diagnóstico recomendado pelo Ministério } \\
\text { da Saúde do Brasil e padronizado pela } \\
\text { OMS. }\end{array}$ & $\begin{array}{c}\text { Melhor conhecimento sobre a } \\
\text { doença e das drogas utilizadas no } \\
\text { tratamento. }\end{array}$ & $\begin{array}{l}\text { Longa duração do tratamento; reações } \\
\text { inflamatórias e de hipersensibilidade } \\
\text { causadas pelo tratamento. }\end{array}$ \\
\hline $\begin{array}{l}\text { Rocha TMS } \\
\text { (2010) }\end{array}$ & $\begin{array}{l}\text { Intervenções breves para } \\
\text { adesão ao tratamento } \\
\text { dos Portadores de } \\
\text { hanseníase que fazem } \\
\text { uso Abusivo de bebida } \\
\text { alcoólica }\end{array}$ & $\begin{array}{l}\text { Classificação do Ministério da Saúde: } \\
\text { Paucibacilar-PB e Multibacilar-MB. }\end{array}$ & $\begin{array}{l}\text { Implantação e implementação de } \\
\text { medidas de rastreamento dos } \\
\text { clientes que abusam da bebida } \\
\text { alcoólica, a fim de intervir junto aos } \\
\text { mesmos na adesão ao tratamento, } \\
\text { na redução do abandono e na } \\
\text { prevenção dos malefícios causados } \\
\text { pelo uso abusivo do álcool. }\end{array}$ & $\begin{array}{c}\text { Alcoolismo e ausência de medidas de } \\
\text { rastreamento e intervenções junto aos } \\
\text { clientes que tem comportamento de } \\
\text { risco. Uso do álcool, que é } \\
\text { subestimado pela equipe } \\
\text { multidisciplinar do Programa de } \\
\text { Hanseníase. }\end{array}$ \\
\hline $\begin{array}{l}\text { Silva NDSH, et } \\
\text { al. (2010) }\end{array}$ & $\begin{array}{c}\text { Diagnóstico de } \\
\text { frequência e adesão das } \\
\text { mulheres ao tratamento } \\
\text { da hanseníase no } \\
\text { Maranhão }\end{array}$ & $\begin{array}{c}\text { Indeterminada (I), Tuberculóide }(T) \text {, } \\
\text { Dimorfa }(D) \text { e Virchowiana }(V) \text { De acordo } \\
\text { com o número de lesões, em: } \\
\text { Paucibacilares (PB = I e T, até cinco } \\
\text { lesões) e Multibacilares (MB = D e V, } \\
\text { acima de cinco lesões) }\end{array}$ & $\begin{array}{c}\text { Apoio da família e da equipe de } \\
\text { saúde mostrou-se relevante para } \\
\text { aceitação da doença e adesão ao } \\
\text { tratamento. }\end{array}$ & $\begin{array}{l}\text { Ausência do assistente social na } \\
\text { equipe de prestação de serviços, a } \\
\text { baixa escolaridade dos pacientes. } \\
\text { Complexidade das orientações } \\
\text { fornecidas ao doente. Sentimento de } \\
\text { impotência em face da saúde. }\end{array}$ \\
\hline $\begin{array}{l}\text { Veras LMC } \\
\quad(2017)\end{array}$ & $\begin{array}{c}\text { A influência da } \\
\text { religiosidade na } \\
\text { aceitação e adesão ao } \\
\text { tratamento de pacientes } \\
\text { com hanseníase }\end{array}$ & $\begin{array}{l}\text { Classificação do Ministério da Saúde: } \\
\text { Paucibacilar-PB e Multibacilar-MB. }\end{array}$ & $\begin{array}{l}\text { A religiosidade interfere de forma } \\
\text { positiva a manutenção da } \\
\text { poliquimioterapia, durante o período } \\
\text { de tratamento. }\end{array}$ & $\begin{array}{l}\text { O preconceito ainda é muito presente } \\
\text { no momento do diagnóstico, implicando } \\
\text { em comprometimento do tratamento. }\end{array}$ \\
\hline
\end{tabular}

Fonte: Saraiva ER, et al., 2020. 
A hanseníase é uma doença infectocontagiosa que tem como agente causador o Mycobacterium leprae (LAMANO LR, 2013), o agente etiológico foi descoberto no ano de 1873, pelo médico norueguês Amaneur Hansen, por isso também é conhecida como bacilo de Hansen (SILVA JUNIOR GB, et al., 2015). O Mycobacterium leprae pertence ao filo Actinobacteria e ao gênero Mycobacterium, possuindo em seu genoma um alto conteúdo de nucleotídeos de citosina e guanina (conteúdo $\mathrm{G}+\mathrm{C}$ ). Estes microrganismos ocorrem como bastonetes retos ou ligeiramente curvados, embora também possam aparecer em forma de hifas que se fragmentam em elementos cocóides ou em bastões (TORTORA GJ, et al., 2005).

A transmissão ocorre de forma direta, por meio das vias aéreas superiores (mucosa nasal e orofaringe), em que uma pessoa doente com a forma MB infecta outras pessoas ao eliminar o bacilo no meio externo (SANTOS KCB, et al., 2019). Diante disso, verifica-se a relevância de monitorar os contatos domiciliares a fim de evitar um maior contágio da doença, uma vez que, o ambiente domiciliar é descrito pelo Ministério da Saúde como importante espaço de transmissão, sendo a investigação epidemiológica fundamental na descoberta de casos entre aqueles que convivem ou conviveram com o doente, tornando-se uma excelente estratégia para a redução da carga bacteriana (BRASIL MS, 2016).

A doença manifesta-se através de sinais e sintomas dermatoneurológicos. O tropismo do bacilo por nervos periféricos é responsável por desordens neurológicas que podem resultar em incapacidades físicas e deformidades (BRASIL MS, 2016). Nesse sentido, quanto mais precoce a doença for detectada e o tratamento for iniciado, menores serão as sequelas adquiridas, corroborando assim para uma melhoria na qualidade de vida do indivíduo.

Quanto ao diagnóstico identificou-se que na maioria das publicações analisadas prevaleceu o preconizado pelo Ministério da Saúde, o qual é dividido em Paucibacilar (PB) e Multibacilar (MB). Em dois trabalhos foi relatado também a Classificação de Madri, que pode ser subdivida em indeterminada (I), dimorfa (D), tuberculoide $(\mathrm{T})$ e virchowiana $(\mathrm{V})$.

Em relação à classificação, Araujo MG (2003) enfatiza que para classificar de maneira completa a Hanseníase são necessários dados provenientes da clínica, da histopatologia, da baciloscopia e do teste da lepromina (Reação de Mitsuda). Apesar das possibilidades a diagnósticas disponíveis e da efetividade do tratamento, estudos como o de Barbosa Júnior AA (1998) e Alves JR (2004), evidenciam dificuldade para se estabelecer o diagnóstico da Hanseníase e sua classificação, com discordância entre o diagnóstico clínico e o achado histopatológico.

Ao abordar a questão da importância do diagnóstico da hanseníase, Araújo MM, et al. (2014) revelam que a descoberta precoce e a detecção dos casos, contribuem positivamente no sentido de coibir a disseminação do círculo transmissor da doença. Além disso, os dados obtidos evidenciaram um número razoável de diagnósticos em fases iniciais da doença.

O estudo de Pereira Junior FACP (2011) destaca que o êxito no tratamento depende de um diagnóstico correto e de uma orientação adequada pelo profissional da saúde informando sobre a administração e regularidade do uso dos medicamentos. Portanto, o diagnóstico correto e o tratamento adequado e precoce são de grande valor para a prevenção de incapacidades, principalmente para evitar os danos neurais. Neste sentido, Abraçado MSF, et al. (2015) destacam que um diagnóstico tardio e tratamento inadequado podem resultar em incapacidade física, principal sequela da hanseníase. Além disso, podem desencadear episódios reacionais hansênicos sucessivos (reentrantes), os quais são respostas exacerbadas do sistema imunológico ao agente agressor.

Em relação a anuência ao trabalho feito pelo MSF, et al. (2015) constatou maior tendência à aceitação de pacientes com formas multibacilares, com um período de diagnóstico de hanseníase maior que um ano, tipo reacional $R R$ e neurites. Este direcionamento constitui um fator positivo para a unidade em que foi realizado o estudo, uma vez que o tratamento multibacilar é extenso, podendo haver efeitos colaterais, já que a quantidade de drogas utilizadas é maior. Os mesmos autores ainda chamam atenção para a influência dos fatores sociodemográficos na adesão ao tratamento das reações hansênicas, sendo que foi observado que pacientes com maior faixa de renda e nível de escolaridade, aderiram mais ao tratamento do que pacientes que possuem maior vulnerabilidade social. 
O estudo de Cunha MHCM, et al. (2013) revelou uma maior tendência de adesão em pacientes do sexo feminino portadoras das formas clínicas paucibacilar. Além disso, sugerem como medida de incentivo a adesão dos pacientes o desenvolvimento de estratégias de educação em saúde em nível individual ou até mesmo com a formação de um grupo de autocuidados, no sentido de reforçar a necessidade de um tratamento sério por parte do paciente e de acompanhamento contínuo.

Luna IT, et al. (2010) corroboram com os resultados de Cunha MHCM, et al (2013) e ressaltam que para uma assistência holística, os serviços em saúde devem conhecer as atividades de vida dos portadores de hanseníase para direcionar as ações voltadas para essa clientela. Fazendo-se necessário a dissipação dos preceitos e estigmas, contribuindo para a melhoria da qualidade no atendimento oferecido a estes indivíduos e melhorando assim a adesão ao tratamento.

Um dos problemas que dificulta a adesão ao tratamento é a ingestão de bebidas alcoólicas. Diante disso, Rocha TMS (2010) aborda em seu trabalho que foram a implantadas medidas de rastreamento dos clientes que fazem uso abusivo de bebida alcoólica, a fim de intervir junto aos mesmos na continuidade do tratamento, na redução do abandono e na prevenção dos malefícios causados.

Dessa forma, foram realizadas capacitações dos profissionais de saúde que atuam no local em que foi aplicada a pesquisa, a fim de dar cumprimento à Política Nacional Sobre o Álcool, prevista no Decreto no 6.117, de 22 de maio de 2007, que preconiza maior engajamento da saúde às estratégias da atenção primária junto à rede do próprio Sistema Único de Saúde (SUS).

Além disso, o papel da religiosidade na influência para a adesão ao tratamento foi destacado por Veras LMC (2017), pois segundo os sujeitos da pesquisa, os benefícios da religiosidade como um auxiliar no processo de reinserção social, da aceitação familiar, dando suporte espiritual e encorajamento pacientes em tratamento para hanseníase. Nesse mesmo sentido, Moreira AJ, et al. (2014) demonstram que muitos estudos apontam, em seus resultados, que maiores níveis de envolvimento religioso estão associados positivamente a indicadores de bem-estar psicológico, como satisfação com a vida, afeto positivo e moral elevado, felicidade, melhor saúde física e mental.

Em relação ao abandono do tratamento, Abraçado MSF, et al. (2015) destacam em seu estudo que a análise da influência dos fatores sociodemográficos mostrou que o sexo masculino tem três vezes mais chances de não aderir ao tratamento do que o feminino. Araújo MM, et al. (2014) também identificaram fatores sociodemográficos que influenciaram no abandono do tratamento, constatando que a maioria dos pacientes que interromperam o tratamento eram do gênero masculino, de raça parda, em idade produtiva, com baixa escolaridade, no início ou metade do tratamento.

Os autores Abraçado MSF, et al. (2015) também relataram que os pacientes, em sua maioria, possuíam baixa escolaridade. Este fator deve ser observado com atenção no momento de verbalizar orientações e esclarecimentos a respeito da hanseníase e das reações hansênicas.

Estudos realizados na Índia e outros no Brasil, como o de Aquino MAV, et al. (2003), correlacionam a importância da educação básica e a melhoria do nível de compreensão do indivíduo acerca da doença e ressaltam este fator como crucial para a baixa adesão ao tratamento hansênico.

Os pacientes multibacilares apresentaram menor adesão ao tratamento, além de possuírem um maior número de contatos intradomiciliares, evidenciando maior risco de transmissão. A não adesão ao tratamento deve-se a outros fatores, que podem ser diretamente relacionados aos indivíduos, ou então, referentes aos serviços de saúde pelos quais são assistidos (ARAÚJO MM, et al., 2014).

O estudo de Pereira Junior FACP (2011) traz como possíveis motivos para o abandono do tratamento a distância da casa do doente para a unidade de saúde, a duração do tratamento, que dependendo do tipo de hanseníase pode levar de seis a oito meses de forma contínua e diária e por último as reações hansênicas, que embora bastante raras acometem alguns indivíduos durante o tratamento, fazendo com que estes interrompam ou abandonem. 
A prestação de informações incompletas pela equipe de saúde é citada por Luna IT, et al. (2010) como um fator que favorece a baixa adesão ao tratamento. Portanto, torna-se necessária a construção de uma comunicação sob diversos prismas, efetivando uma maior interação entre profissional e paciente.

O abandono do tratamento é correlacionado por Cunha MHCM, et al. (2013) juntamente com as consequências e interferências das reações hansênicas para o paciente e os autores apontaram como principais causas da não adesão: a dor, o desconforto físico, a alteração na aparência física e sofrimento, além da grande dificuldade ou impossibilidade de executar tarefas domésticas.

Um dos motivos para abandono do tratamento junto aos clientes que tem comportamento de risco é destacado por Rocha TMS (2010), como o uso do álcool é subestimado pela equipe multidisciplinar do Programa de Hanseníase, conforme ficou constatado, apesar deste uso ser um dos fatores de não adesão e abandono do tratamento da doença, com o agravante de que há desinformação a respeito do significado da expressão "bebedor social" que, na maioria das vezes, encobre uma situação de compulsão pela ingestão alcoólica que caracteriza o abusador.

Além disso, Silva NDSH, et al. (2010) também elencam como elemento relevante para que isso ocorra, o sentimento de impotência diante do quadro de saúde pelo qual o paciente com hanseníase se encontra no momento do diagnóstico, que em muitas vezes está em fase avançada. O preconceito é descrito por Veras LMC (2017) como sendo muito frequente no momento do diagnóstico da hanseníase, implicando em comprometimento do tratamento e podendo acarretar no abandono do mesmo. Alguns mitos ainda são observados, entre eles a cultura de separar os utensílios domésticos sendo que um dos maiores desafios para a adesão ao tratamento é o preconceito do próprio paciente no seu domicílio.

Ainda nesse contexto, Paomeira IP, et al. (2012) também abordam a questão da discriminação ao relatar que as pessoas têm um imaginário sobre o contágio que acaba influenciando e atravessando a representação social da hanseníase, por mais que se tenha o discurso técnico-científico da desconstrução disso. Mesmo assim, as pessoas acometidas pela doença sofrem o peso do estigma relacionado à doença.

\section{CONSIDERAÇÕES FINAIS}

A hanseníase ainda constitui um desafio a ser superado pela saúde pública brasileira e o diagnóstico precoce é fundamental na diminuição do processo de transmissibilidade. No que se refere à adesão, ressaltase a importância das ações de educação em saúde, o papel da religiosidade e dos vínculos familiares no sentido de fornecer subsídios para o enfrentamento da doença. Já em relação ao abandono do tratamento, identificou-se que a maioria dos pacientes eram do sexo masculino e com baixo nível de escolaridade. Outro fator relevante está na qualidade das informações repassadas para este público, os quais possuem dificuldade de compreensão. Somado a isso, incluem-se os elementos físicos como dor, alteração na aparência, a discriminação e impotência sentida diante do problema de saúde que fazem com que o paciente abandone o tratamento.

\section{REFERÊNCIAS}

1. ABRACADO MFS, et al. Adesão ao tratamento de hanseníase em pacientes com episódios reacionais hansênicos em uma unidade de referência. RevPan-AmazSaude, Ananindeua, 2015; 6(2): 23-28.

2. AQUINO MAV, et al. Perfil dos pacientes com hanseníase em área hiperendêmica do Estado do Maranhão, 1991 1995. Cad. Saude Publica. 2003; 19(1):119-25.

3. ARAÚJO MG. Hanseníase no Brasil. Rev Soc Bras Med Trop. 2003; 3(36): 373-382.

4. ARAÚJO MM, et al. Perfil clínico-epidemiológico de pacientes que abandonaram o tratamento de hanseníase. Hansen. Int. 2014; 39(2): 55-63.

5. ARAUJO AERA, et al . Complicações neurais e incapacidades em hanseníase em capital do nordeste brasileiro com alta endemicidade. Rev. bras. epidemiol. São Paulo, 2014; 17(4): 899910.

6. BOECHAT N, PINHEIRO LCS. A hanseníase e a sua quimioterapia. Rev. virtual quim. 2012; 4(3): 247-256.

7. BRASIL MS. Secretaria de Vigilância em Saúde. Departamento de Vigilância Epidemiológica. Programa Nacional de Controle da Hanseníase. Manual de Prevenção de Incapacidade: cadernos de prevenção e reabilitação em hanseníase. Brasília: Ministério da Saúde, 2008; 140p. 
8. BRASIL MS. Secretaria de Vigilância em Saúde, Departamento de Vigilância das Doenças Transmissíveis. Diretrizes para vigilância, atenção e eliminação da Hanseníase como problema de saúde pública: manual técnico-operacional. Brasília: Ministério da Saúde, 2016; 58p.

9. CARVALHO KA. Discussões em torno da reconstrução do significado da lepra no período pós-sulfônico, Minas Gerais, na década de 1950. Rev. História, Ciências, Saúde - Manguinhos. 2015; 22(2): 541-557.

10. CUNHA MHCM, et al. Episódios reacionais hansênicos: estudo de fatores relacionados com adesão ao tratamento em uma unidade de referência. Hansen Int. 2013; 38(1-2): 61-67.

11. GALVAO TF, PEREIRA MG. Revisões sistemáticas da literatura: passos para sua elaboração. Epidemiol. Serv. Saúde, Brasília. 2014; 23(1): 183-184.

12. LAMANO LR. Estudo da diversidade genética do Mycobacterium leprae em pacientes com hanseníase no município de Rondonópolis (MT): uma contribuição para o melhor entendimento da sua transmissão. Dissertação (mestrado) Universidade Estadual Paulista Júlio de Mesquita Filho, Faculdade de Medicina de Botucatu, Botucatu, São Paulo, 2013; 89p.

13. LASTORIA JC, ABREU MAMM. Leprosy: a review of laboratory and therapeutic aspects - Part 2. An. Bras. Dermatol., Rio de Janeiro. 2014; 89(3): 389-401.

14. LOPES VAS, RANGEL EM. Hanseníase e vulnerabilidade social: uma análise do perfil socioeconômico de usuários em tratamento irregular. Saúde em Debate. 2014; 38(103): 817-829.

15. LUNA IT, et al. Adesão ao tratamento da Hanseníase: dificuldades inerentes aos portadores. Rev. bras. enferm., Brasília. 2010; 63(6): 983-990.

16. MELAO S, et al. Perfil epidemiológico dos pacientes com hanseníase no extremo sul de Santa Catarina, no período de 2001 a 2007. Rev. Soc. Bras. Med. Trop., Uberaba. 2011; 44(1): 79-84.

17. MOREIRA AJ, et al. Ação educativa sobre hanseníase na população usuária das unidades básicas de saúde de Uberaba - MG. Saúde em debate, Rio de Janeiro. 2014; 38(101): 234-243.

18. PAZ BL, et al. Hanseníase e os desafios para sua erradicação: casos notificados em um município no Ceará. Rev. Interdisciplinar. 2018; 11(2): 37-46.

19. PAOMEIRA IP, et al. Quando o preconceito marca mais que a doença. Revista Tempus Actas de Saúde Coletiva. 2012; (6)3: 187-199.

20. PEREIRA JUNIOR FAC. Motivos do abandono ou interrupção do tratamento da hanseníase: uma revisão sistemática da literatura. TCC (Especialização de Sistema e serviços de saúde) - Centro de Pesquisas Aggeu Magalhães, Recife, $2011 ; 24 p$.

21. PEREIRA SVM, et al. Avaliação da Hanseníase: Relato de experiência de acadêmicos de enfermagem. Revista Brasileira de Enfermagem. Brasília. 2008; 61(esp): 774-80.

22. PINHEIRO MGC, et al. Compreendendo a "alta em hanseníase": uma análise de conceito. Rev.Gaúcha Enferm.,Porto Alegre. 2017; 38(4): 63-90.

23. PINHEIRO MMO. Hanseníase em registro ativo no município de Passos, MG - Brasil. 2006. Dissertação (Mestrado em Promoção da Saúde) - Universidade de Franca, Franca, São Paulo, 2007; 60p.

24. RAMOS JMH, SOUTO FJD. Incapacidade pós-tratamento em pacientes hansenianos em Várzea Grande, Estado de Mato Grosso. Rev. Soc. Bras. Med. Trop. 2010; 43(3): 293-7.

25. RIBEIRO MDA. Estudo epidemiológico da hanseníase no Brasil: reflexão sobre as metas de eliminação. Revista Panamericana de Salud Pública[online]. 2018; 42(2): 1680-5348.

26. ROCHA TMS. Intervenções breves para adesão ao tratamento dos portadores de hanseníase que fazem uso abusivo de bebida alcoólica. 2010. DISSERTAÇÃO (Mestrado em Enfermagem). Universidade Federal do Rio de Janeiro, 2010; $151 \mathrm{p}$.

27. ROSA SPS, et al. Incidência de hanseníase na Paraíba entre os anos de 2010 a 2014. Revista Brasileira de Educação e Saúde. 2016; 6(4): 22-26.

28. SANTOS KCB, et al. Estratégias de controle e vigilância de contatos de hanseníase: revisão integrativa. Saúde debate, Rio de Janeiro. 2019; 43(121): 576-591.

29. SILVA JUNIOR GB, et al. Leprosy nephropathy: a review of clinical and histopathological features. Revista do Instituto de Medicina Tropical de São Paulo, São Paulo. 2015; 57(1): 15-20.

30. SILVA DRX, et al. Hanseníase, condições sociais e desmatamento na Amazônia brasileira. Revista Panamericana de Saúde Pública. 2010; 27(4): 268-75.

31. SILVA NDSH, et al. Diagnóstico de frequência e adesão das mulheres ao tratamento da hanseníase no Maranhão. Infarma - Ciências Farmacêuticas. 2012; 22(7/8): 2318-9312.

32. SOUZA MT, et al. Integrative review: what is it? How to do it? Einstein, São Paulo. 2010; 8(1):102-6.

33. TORTORA GJ, et al. Microbiologia. Traduzido de Microbiology: An Introduction. 8 ed. Porto Alegre: Artmed, 2005; $632 p$.

34. VELÔSO DS, et al. Perfil Clínico Epidemiológico da Hanseníase: Uma Revisão Integrativa. Revista Eletrônica Acervo Saúde/Electronic Journal Collection Health. 2018; 10(1): 1429-1437.

35. VERAS LMC. A influência da religiosidade na aceitação e adesão ao tratamento de pacientes com hanseníase. Dissertação (Mestrado Profissional em Saúde da Família). Centro Universitário UNINOVAFAPI, Teresina, 2017; 73p. 